\title{
ON STABILITY OF ADDITIVE MAPPINGS
}

\section{ZBIGNIEW GAJDA}

Institute of Mathematics

Silesian University

Bankowa 14

40-007 Katowice

Poland

(Received January 26, 1990)

\begin{abstract}
In this paper we answer a question of Th. M. Rassias concerning an extension of validity of his result proved in [3].
\end{abstract}

KEY WORDS AND PHRASES. Additive mappings, linear mappings, Banach spaces, stability. 1980 AMS SUBJECT CLASSIFICATION CODES. Primary 39B70, Secondary 39C05.

\section{INTRODUCTION.}

In connection with a problem posed by Ulam (cf. [5]; see also [2]) Th. M. Rassias [3] proved the following theorem on stability of linear mappings in Banach spaces.

THEOREM 1. (see [3]) Let $E_{1}$ and $E_{2}$ be two (real) Banach spaces and let $f: E_{1} \rightarrow E_{2}$ be a mapping such that for each fixed $x \in E_{1}$ the transformation $\mathbb{R} \ni t \rightarrow f(t x)$ is continuous. Moreover, assume that there exist $\varepsilon \in[0, \infty)$ and $p \varepsilon[0,1\}$ such that

$$
\|f(x+y)-f(x)-f(x)\| \leq \varepsilon\left(\|x\|^{p}+\|y\|^{p}\right)
$$

for all $x, y \varepsilon E_{1}$. Then there exists a unique linear mapping $T: E_{1} \rightarrow E_{2}$ such that

$$
\|f(x)-T(x)\| \leq \delta\|x\|^{p}
$$

for all $x \varepsilon E_{1}$, where $\delta:=\frac{2 \varepsilon}{2-2^{p}}$.

As was mentioned by Th. M. Rassias [4], the proof presented in [3] reveals that, in fact, it works for every $p$ from the interval $(-\infty, 1)$ and, therefore, the theorem holds true for all such $p$ 's. It is also readily seen that the only purpose of assuming that all the transformations of the form $t \rightarrow f(t x)$ are continuous is to guarantee the real homogeneity of the mapping $T$. Without this assumption one can show that $f$ is approximated by an additive mapping $T$ which means that $T$ satisfies the following equation

$$
T(x+y)=T(x)+T(y)
$$

for all $x, y \varepsilon E_{1}$. Finally, it should be noticed that the completeness of the space $E_{1}$ may be removed from the assumptions of Theorem 1. However, there is still one non-trivial (as it seems) question concerning a possible extension of the range of validity of Theorem 1 . Namely, one can ask whether the same result holds true under the hypothesis that $p$ is taken from the interval $[1, \infty)$ (obviously in this case the constant $\delta$ should have been defined in a different manner). Such a 
problem was raised by Th. M. Rassias during the 27th International Symposium on Functional Equations which was held in Bielsko-Biala, Katowice and Krokow in August 1989. The goal of the present note is to give a complete solution to this problem.

\section{MAIN RESULTS.}

First, let us realize why the proof of Theorem 1 in its original form (see [3]) does not work for $p \geq 1$. The fundamental role in this proof is played by the sequence

$$
\left\{\frac{1}{2^{n}} f\left(2^{n} x\right): n \varepsilon \mathbb{N}\right\}
$$

which, under the assumptions of Theorem 1 (in fact as long as $p \varepsilon(-\infty, 1)$ ) is convergent for each fixed $x \varepsilon E_{1}$. Then $T: E_{1} \rightarrow E_{2}$ defined by the formula

$$
T(x):=\lim _{n} \frac{1}{2^{n}} f\left(2^{n} x\right), \quad x \varepsilon E_{1}
$$

is the desired linear mapping approximating $f$. The argument ensuring the convergence of sequence (2.1) is no longer valid when $p$ becomes greater or equal to 1 , so in order to carry the proof over to this case, one has to change the argument itself or the definition of the mapping $T$. It turns out that, for $p>1$, the latter modification of the proof is possible. As a result we obtain the following extension of Theorem 1 :

THEOREM 2. Let $E_{1}$ and $E_{2}$ be two (real) normed linear spaces and assume that $E_{2}$ is complete. Let $f: E_{1} \rightarrow E_{2}$ be a mapping for which there exist two constants $\varepsilon \in[0, \infty)$ and $p \in \mathbb{R} \backslash\{1\}$ such that

$$
\|f(x+y)-f(x)-f(y)\| \leq \varepsilon\left(\|x\|^{p}+\|y\|^{p}\right)
$$

for all $x, y \varepsilon E_{1}$. Then there exists a unique additive mapping $T: E_{1} \rightarrow E_{2}$ such that

$$
\|f(x)-T(x)\| \leq \delta\|x\|^{p}
$$

for all $x \varepsilon E_{1}$, where

$$
\delta= \begin{cases}\frac{2 \varepsilon}{2-2^{p}} & \text { for } p<1 \\ \frac{2 \varepsilon}{2^{p}-2} & \text { for } p>1\end{cases}
$$

Moreover, is for each $x \in E_{1}$ the transformation $\mathbb{R} \ni t \rightarrow f(t x)$ is continuous, then the mapping $T$ is linear.

PROOF. In view of what has been said so far, it remains to consider the case $p>1$. The main innovation in comparison with the case $p<1$ consists in defining the mapping $T$ by the formula

$$
T(x):=\lim _{n \rightarrow \infty} 2^{n} f\left(\frac{x}{2^{n}}\right), \quad x \varepsilon E_{1}
$$

instead of (2.2). Obviously, one has to verify the convergence of the sequence occurring on the right-hand side of (2.5).

Putting $\frac{x}{2}$ in place of $x$ and $y$ in inequality (2.3), we obtain

$$
\left\|f(x)-2 f\left(\frac{x}{2}\right)\right\| \leq 2 \varepsilon\left\|\frac{x}{2}\right\|^{p}=2^{1-p} \varepsilon\|x\|^{p}
$$

for all $x \in E_{1}$. Hence for each $n \varepsilon \mathbb{N}$ and every $x \in E_{1}$, we have

$$
\begin{aligned}
\left\|f(x)-2^{n} f\left(\frac{x}{2^{n}}\right)\right\| & \leq\left\|f(x)-2 f\left(\frac{x}{2}\right)\right\|+2\left\|f\left(\frac{x}{2}\right)-2 f\left(\frac{x}{2^{2}}\right)\right\|+\ldots+2^{n-1}\left\|f\left(\frac{x}{2^{n-1}}\right)-2 f\left(\frac{x}{2^{n}}\right)\right\| \\
& \leq 2^{1-p} \varepsilon\|x\|^{p}+2 \cdot 2^{1-p} \varepsilon\left\|\frac{x}{2}\right\|^{p}+\ldots+2^{n-1} \cdot 2^{1-p} \varepsilon\left\|\frac{x}{2^{n-1}}\right\|^{p} \\
& =\left(2^{1-p}+2^{2(1-p)}+\ldots+2^{n(1-p)}\right) \varepsilon\|x\|^{p}
\end{aligned}
$$




$$
\leq \delta\|x\|^{p}
$$

where $\delta$ is the sum of the following convergent series:

$$
\sum_{n=1}^{\infty} 2^{n(1-p)} \varepsilon=\frac{2 \varepsilon}{2^{p}-2} .
$$

Now, fix an $x \varepsilon E_{1}$ and choose arbitrary $m, n \varepsilon \mathbb{N}$ such that $m>n$. Then

$$
\begin{aligned}
\left\|2^{m} f\left(\frac{x}{2^{m}}\right)-2^{n} f\left(\frac{x}{2^{n}}\right)\right\| & =2^{n}\left\|2^{m-n} f\left(\frac{1}{2^{m-n}} \cdot \frac{x}{2^{n}}\right)-f\left(\frac{x}{2^{n}}\right)\right\| \\
& \leq 2^{n} \delta\left\|\frac{x}{2^{n}}\right\|^{p}=2^{n(1-p)} \delta\|x\|^{p},
\end{aligned}
$$

which becomes arbitrarily small as $n \rightarrow \infty$. On account of the completeness of the space $E_{2}$, this implies that the sequence $\left\{2^{n} f\left(\frac{x}{2^{n}}\right): n \varepsilon \mathbb{N}\right\}$ is convergent for each $x \in E_{1}$. Thus $T$ is correctly defined by (2.5). Moreover, it satisfies condition (2.4) which results on letting $n \rightarrow \infty$ in (2.6).

Finally, replacing $x$ by $\frac{x}{2^{n}}$ and $y$ by $\frac{y}{2^{n}}$ in (2.3) and then multiplying both sides of the resulting inequality by $2^{n}$, we get

$$
\left\|2^{n} f\left(\frac{x+y}{2^{n}}\right)-2^{n} f\left(\frac{x}{2^{n}}\right)-2^{n} f\left(\frac{y}{2^{n}}\right)\right\| \leq 2^{n(1-p)} \varepsilon\left(\|x\|^{p}+\|y\|^{p}\right),
$$

for $x, y \in E_{1}$. Since the right-hand side of this inequality tends to zero as $n \rightarrow \infty$, it becomes apparent that the mapping $T$ defined by (2.5) is additive.

The proof of the homogeneity of $T$ (under the supplementary assumption that $t \rightarrow f(t x)$ is continuous for each $x \in E_{1}$ ) needs no essential alterations in comparison with the case $p<1$. It is also clear what has to be changed in the proof of the uniqueness of $T$.

Theorem 2 leaves the case $p=1$ undecided. This is not a mere coincidence. It turns out that 1 is the only critical value of $p$ to which Theorem 2 can not be extended. In fact, we shall show that $\varepsilon>0$ one can find a function $f: \mathbb{R} \rightarrow \mathbb{R}$ such that

$$
|f(x+y)-f(x)-f(y)| \leq \varepsilon(|x|+|y|)
$$

for all $x, y \varepsilon R$, but, at the same time, there is no constant $\delta \in[0, \infty)$ and no additive function $T: \mathbf{R} \rightarrow \mathbf{R}$ satisfying the condition

$$
|f(x)-T(x)| \leq \delta|x| \quad \text { for all } x \varepsilon \mathbb{R} .
$$

This singularity is illustrated by the following:

EXAMPLE. Fix $\varepsilon>0$ and put $\mu:=\frac{\varepsilon}{6}$. First we define a function $\phi: \mathbb{R} \rightarrow \mathbb{R}$ by

$$
\phi(x):= \begin{cases}\mu & \text { for } x \varepsilon[1, \infty), \\ \mu x & \text { for } x \varepsilon(-1,1), \\ -\mu & \text { for } x \varepsilon(-\infty,-1] .\end{cases}
$$

Evidently, $\phi$ is continuous and $|\phi(x)| \leq \mu$ for all $x \varepsilon \mathbb{R}$. Therefore, a function $f: \mathbb{R} \rightarrow \boldsymbol{R}$ is correctly defined by the formula

$$
f(x):=\sum_{n=0}^{\infty} \frac{\phi\left(2^{n} x\right)}{2^{n}}, \quad x \in \mathbf{R} .
$$

Since $f$ is defined by means of a uniformly convergent series of continuous functions, $f$ itself is continuous. Moreover,

$$
|f(x)| \leq \sum_{n=0}^{\infty} \frac{\mu}{2^{n}}=2 \mu, \quad x \in \mathbf{R} .
$$

We are going to show that $f$ satisfies (2.7). 
If $x=y=0$, then (2.7) is trivially fulfilled. Next assume that $0<|x|+|y|<1$. Then there exists an $N \varepsilon \mathbb{N}$ such that

$$
2^{N} \leq|x|+|y|<\frac{1}{2^{N-1}}
$$

Hence, $\quad\left|2^{N-1} x\right|<1, \quad\left|2^{N-1} y\right|<1$ and $\quad\left|2^{N-1}(x+y)\right| \leq 2^{N-1}(|x|+|y|)<1$, which implies that for each $n \in\{0,1, \ldots, N-1\}$ the numbers $2^{n} x, 2^{n} y$ and $2^{n}(x+y)$ remain in the interval $(-1,1)$. Since $\phi$ is linear on this interval, we infer that

$$
\phi\left(2^{n}(x+y)\right)-\phi\left(2^{n} x\right)-\phi\left(2^{n} y\right)=0
$$

for $n=0,1, \ldots, N-1 . \quad$ As a result, we get

$$
\begin{aligned}
\frac{|f(x+y)-f(x)-f(x)|}{(|x|+|y|)} & \leq \sum_{n=N}^{\infty} \frac{\left|\phi\left(2^{n}(x+y)\right)-\phi\left(2^{n} x\right)-\phi\left(2^{n} y\right)\right|}{2^{n}(|x|+|y|)} \\
& \leq \sum_{k=0}^{\infty} \frac{3 \mu}{2^{k_{2}}(|x|+|y|)} \leq \sum_{k=0}^{\infty} \frac{3 \mu}{2^{k}}=6 \mu=\varepsilon .
\end{aligned}
$$

Finally, assume that $|x|+|y| \geq 1$. Then merely by virtue of the boundedness of $f$ we have

$$
\frac{|f(x+y)-f(x)-f(Y)|}{|x|+|y|} \leq 6 \mu=\varepsilon .
$$

Thus we conclude that $f$ satisfies (2.7) for all real $x$ and $y$.

Now, contrary to what we claim, suppose that there exist a $\delta \in[0, \infty)$ and an additive function $T: \mathbb{R} \rightarrow \mathbb{R}$ such that (2.8) holds true. Hence, from the continuity of $f$ it follows that $T$ is bounded on some neighbourhood of zero. Then, by a classical result (see e.g. [1], 2.1.1., Theorem 1) there exists a real constant $c$ such that

$$
T(x)=c x, \quad x \in \mathbb{R}
$$

Hence,

which implies that

$$
|f(x)-c x| \leq \delta|x|, \quad x \in \mathbb{R},
$$

$$
\left|\frac{f(x)}{x}\right| \leq \delta+|c|, \quad x \in \mathbb{R} .
$$

On the other hand, we can choose an $N \varepsilon \mathbb{N}$ so large that $N \mu>\delta+|x|$ : Then picking out an $x$ from the interval $\left(0, \frac{1}{2^{N-1}}\right)$, we have $2^{n} x \in(0,1)$ for each $n \in\{0,1, \ldots, N-1\}$. Consequently, for such an $x$ we have

$$
\frac{f(x)}{x} \geq \sum_{n=0}^{\infty} \frac{\phi\left(2^{n} x\right)}{2^{n} x}=\sum_{n=0}^{\infty} \frac{\mu 2^{n} x}{2^{n} x}=N \mu>\delta+|x|,
$$

which yields a contradiction. Thus the function $f$ provides a good example to the effect that Theorem 2 fails to hold for $p=1$.

\section{REFERENCES}

1. ACZEL, J. Lectures on Functional Equations and their Applications, Academic Press, New York - San Francisco - London, 1966.

2. HYERS, D.H. On the stability of the linear functional equation, Proc. Nat. Acad. Sci., U.S.A., 27 (1941), 222-224.

3. RASSIAS, TH. M. On the stability of the linear mapping in Banach spaces, Proc. Amer. Math. Soc. $\underline{72}$ (1978), 297-300.

4. RASSIAS, TH. M. Communication, 27th International Symposium on Functional Equations, Bielsko-Biala, Katowice, Krokow, Poland, 1989.

5. ULAM, S.M. Problems in modern mathematics, Chapter VI, Science Editions, Wiley, New York, 1960. 


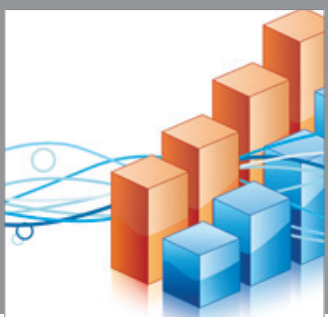

Advances in

Operations Research

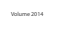

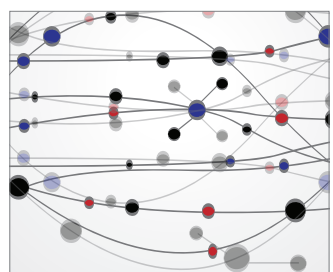

\section{The Scientific} World Journal
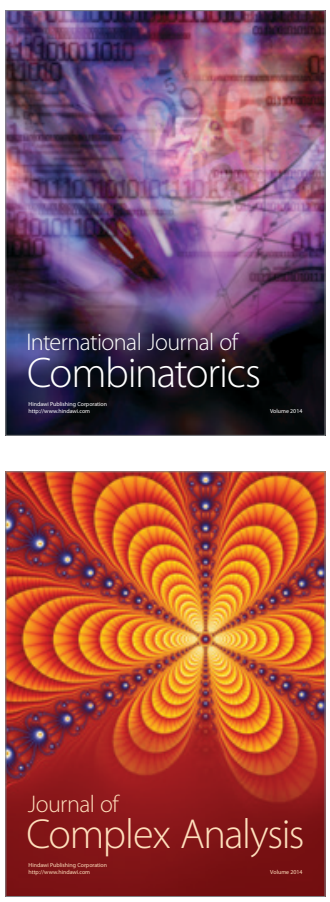

International Journal of

Mathematics and

Mathematical

Sciences
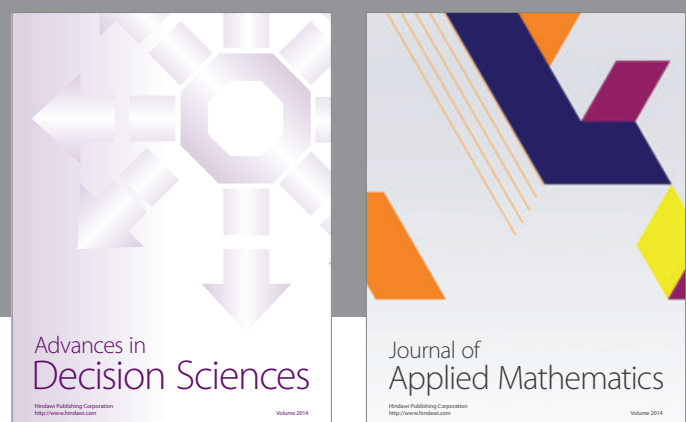

Journal of

Applied Mathematics
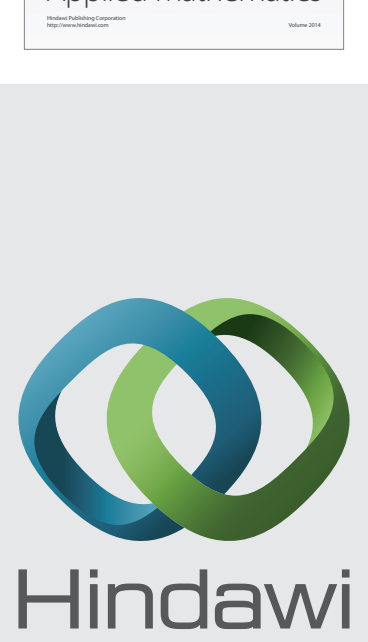

Submit your manuscripts at http://www.hindawi.com
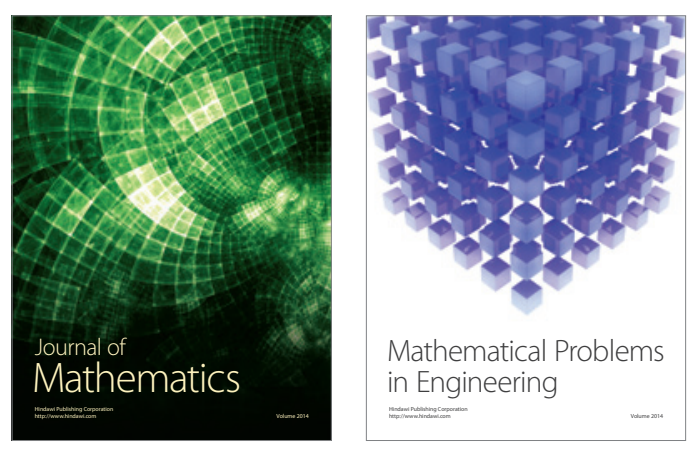

Mathematical Problems in Engineering
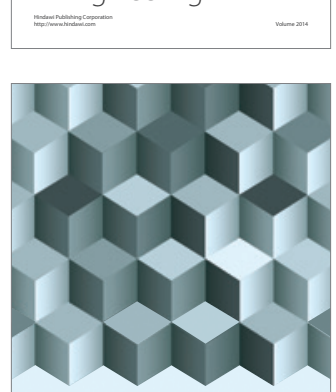

Journal of

Function Spaces
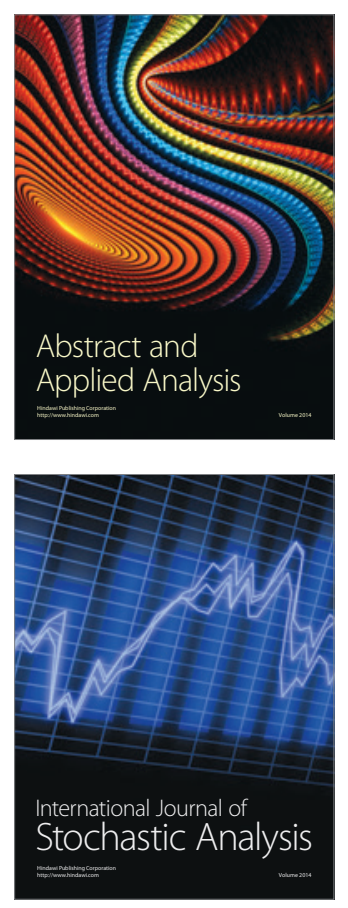

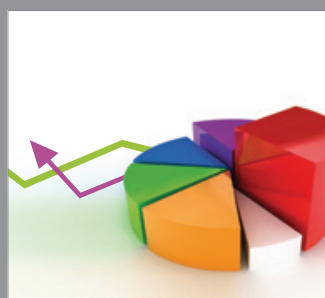

ournal of

Probability and Statistics

Promensencen
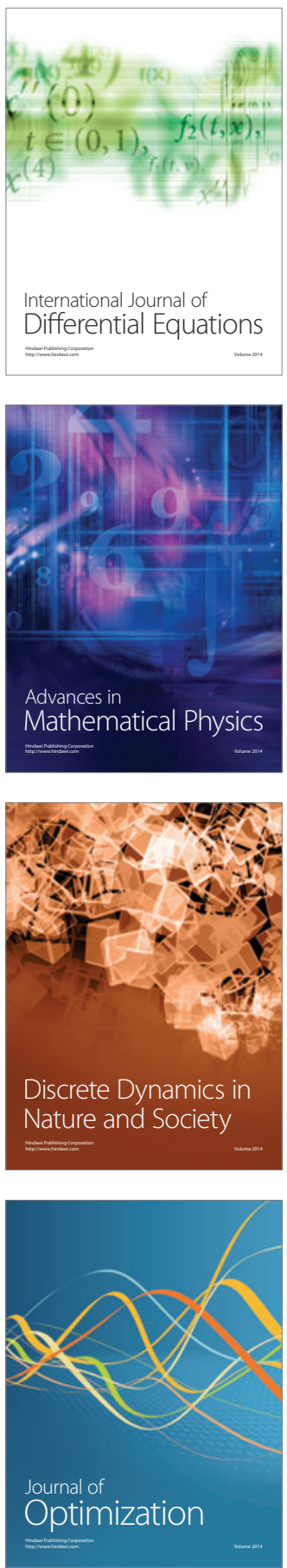